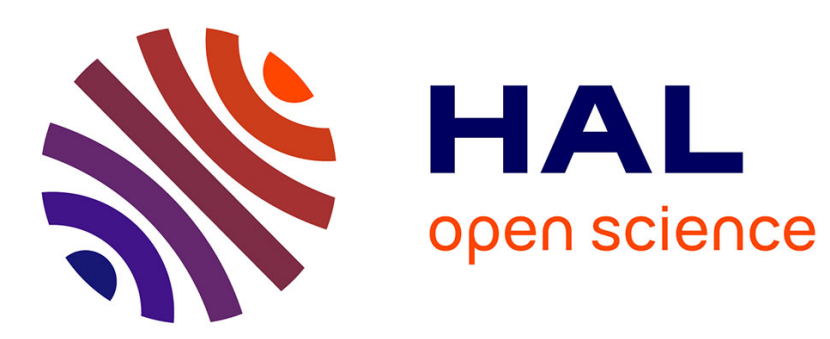

\title{
Dynamics of excitable elements with time-delayed coupling
}

\author{
S. Rüdiger, L. Schimansky-Geier
}

\section{To cite this version:}

S. Rüdiger, L. Schimansky-Geier. Dynamics of excitable elements with time-delayed coupling. Journal of Theoretical Biology, 2009, 259 (1), pp.96. 10.1016/j.jtbi.2009.01.030 . hal-00554571

\section{HAL Id: hal-00554571 \\ https://hal.science/hal-00554571}

Submitted on 11 Jan 2011

HAL is a multi-disciplinary open access archive for the deposit and dissemination of scientific research documents, whether they are published or not. The documents may come from teaching and research institutions in France or abroad, or from public or private research centers.
L'archive ouverte pluridisciplinaire HAL, est destinée au dépôt et à la diffusion de documents scientifiques de niveau recherche, publiés ou non, émanant des établissements d'enseignement et de recherche français ou étrangers, des laboratoires publics ou privés. 


\section{Author's Accepted Manuscript}

Dynamics of excitable elements with time-delayed coupling

S. Rüdiger, L. Schimansky-Geier

PII: $\quad$ S0022-5193(09)00048-4

DOI: $\quad$ doi:10.1016/j.jtbi.2009.01.030

Reference: $\quad$ YJTBI5453

To appear in: $\quad$ Journal of Theoretical Biology

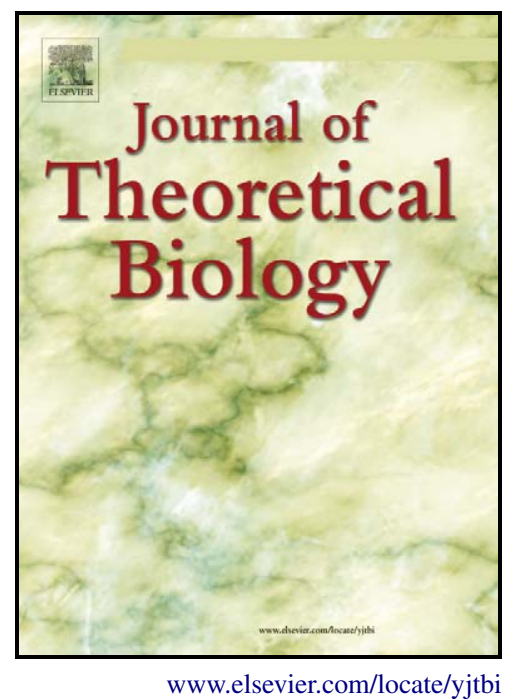

Received date: $\quad 10$ October 2008

Revised date: $\quad 29$ January 2009

Accepted date: $\quad 30$ January 2009

Cite this article as: S. Rüdiger and L. Schimansky-Geier, Dynamics of excitable elements with time-delayed coupling, Journal of Theoretical Biology (2009), doi:10.1016/j.jtbi.2009.01.030

This is a PDF file of an unedited manuscript that has been accepted for publication. As a service to our customers we are providing this early version of the manuscript. The manuscript will undergo copyediting, typesetting, and review of the resulting galley proof before it is published in its final citable form. Please note that during the production process errors may be discovered which could affect the content, and all legal disclaimers that apply to the journal pertain. 


\title{
Dynamics of excitable elements with time-delayed coupling
}

\author{
S. Rüdiger, L. Schimansky-Geier \\ Institut of Physics, Humboldt University, Newtonstr. 15, 12489 Berlin, Germany
}

\begin{abstract}
Motivated by recent experiments on intracellular calcium release we study the effects of different types of coupling on the dynamics of arrays of excitable elements. We intend to find a mechanism, which produces a sustained activity of the elements following a spike. While instantaneous diffusive coupling does not exhibit this property, we show that, for a coupling term with temporal delay, signals from adjacent elements can serve as mutual excitations and thus prolong the duration of the signal. We propose that time delayed coupling is generated by diffusion between isolated clusters of calcium channels. Our model could thus provide an explanation for two different release modes observed in the $\mathrm{Ca}^{2+}$ system.

Key words: intracellular calcium dynamics; puff; FitzHugh-Nagumo model; buffer protein; delay; excitability
\end{abstract}

\section{Introduction}

The dynamics of intracellular calcium is an important biological system, where excitable dynamics are believed to be fundamental. The spatial and temporal signals that are built on concentration changes of $\mathrm{Ca}^{2+}$ are central to many cellular functions ranging from gene-expression to cell death. External signals trigger the generation of $\mathrm{IP}_{3}$, which stimulates receptor channels $\left(\mathrm{IP}_{3} \mathrm{R}\right)$ situated on the membrane of the endoplasmic reticulum (ER). Once stimulated, the channels release $\mathrm{Ca}^{2+}$ from the ER in response to binding of $\mathrm{Ca}^{2+}$ ions to the channel, and are thus subjected to a feedback mechanism (Falcke, 2004). 
This $\mathrm{Ca}^{2+}$ loop, together with the coupling of channels by diffusing calcium, produces complex but controlled spatio-temporal release patterns, allowing the system to relay various signals to cell organelles.

An important aspect of the pattern generation is the fact that in many cells the $\mathrm{IP}_{3} \mathrm{R}$ channels are grouped into clusters of 40-70 channels (Shuai et al., 2007). Opening events can therefore involve single channels, few or all channels in one cluster, or many channels from several clusters. The different release modes are indeed observed experimentally and termed blip, puff (Yao \& Parker, 1995; Callamaras \& Parker, 2000), or global release, respectively. The fact that the different modes result from different channel synchronization strengths was studied in many publications and can be summarized as follows.

In the global mode, clusters of calcium ion channels are strongly coupled and stimulate each other to generate a wave of receptor excitation and calcium release. In the local mode (puffs), only the channels in individual clusters are excited. The latter case can appear due to reduced cooperativity of channels in different clusters. It was found that the addition of an exogenous buffer protein (EGTA) compels the transition from global to local release (Callamaras $\&$ Parker, 2000). The transition can be understood from the fact that additional buffer reduces the spatial extent of calcium domains, thereby decreasing the amount of $\mathrm{Ca}^{2+}$ diffusing to adjacent clusters.

The cooperativity of channels can therefore explain the transition from global to local release, but it will not be the direct focus of this presentation. Instead we here turn to the interesting and not yet understood aspect, that signals decay with different time scales depending on the mode of calcium release (local or global), that is, we study the temporal shape of the release. It was observed that the decay of cluster activity is much faster in the local than in the global mode (Jouaville et al., 1995; Callamaras \& Parker, 2000; Dargan \& Parker, 2003). If the coupling of clusters in the global mode is indeed stronger, one could then say, that the clusters keep each other active and therefore the activity of the channels decays very slowly. In this paper we want to study how exactly this mechanism of mutual excitation could be accomplished. 
To simplify the problem we will not use a detailed model for $\mathrm{Ca}^{2+}$ dynamics. Such detailed models (see, for instance, Rüdiger et al. (2007)) consist of deterministic sets of reaction-diffusion equations for the concentrations of $\mathrm{Ca}^{2+}$ and various buffer proteins coupled to the release of $\mathrm{Ca}^{2+}$ from the channels. The state of each channel (open or closed) is given by a master equation for channel states, which can be solved by a stochastic scheme. The combined dynamics of the model gives rise to concerted openings of channels inside of a cluster. However, for our current purpose it suffices to assume an individual cluster to be one dynamical element. It has been shown by several authors that the collective dynamics of channels in a cluster can be described by FitzHugh-Nagumo-type equations (Meinhold \& Schimansky-Geier, 2002; Li \& Rinzel, 1994), where an activator variable represents, for instance, the calcium concentration and an inhibitor variable stands for the number of inhibited channels. Thul \& Falcke (2004), particularly, showed that deterministic oscillatory local dynamics may be difficult to achieve in realistic models of clusters, given the large local concentrations of calcium in the surrounding of the channels. Consequently, recent models are based on excitability and rely on fluctuations in the channel gating for initiation of release. Since excitable behavior is prototypically modeled by FitzHugh-Nagumo (FHN) equations, we will in this work identify a single cluster with an excitable FHN element. To mimic the coupling of clusters of $\mathrm{Ca}^{2+}$ channels by diffusing $\mathrm{Ca}^{2+}$ we will consider arrays of FHN elements coupled to their nearest neighbors.

The form of this coupling is at the focus of our work. Besides standard diffusive behavior we study temporally delayed coupling and find that only the latter produces distinct local and global relaxation times. Delayed coupling can be expected for the calcium system since clusters are separated by receptor-free regions, where calcium (and calcium bound buffer) diffuses but does not activate further release. Signals from active clusters arrive at neighboring clusters after temporal delay, which is the time the calcium needs to diffuse towards it. Therefore, coupling of excitable elements in the calcium system possesses specific properties different from standard diffusion and may markedly shape 
spatio-temporal patterns.

The FHN model is an exemplary system to describe the dynamics of excitable behavior in neurons. In our study it represents a large class of similar systems, where we expect the delay mechanism to work. We believe that the effect described in this paper is applicable to other systems, such as arrays of coupled neurons (modeled, for instance, by Hodgkin-Huxley dynamics, Keener \& Sneyd (1998)).

\section{An array with diffusive coupling}

We begin with an array of $n$ diffusively coupled FHN elements:

$$
\begin{aligned}
\frac{\partial v_{i}}{\partial t} & =v_{i}\left(1-v_{i}\right)\left(v_{i}-a\right)-w_{i}-w_{0}+\sigma \xi_{i}+c\left(v_{i-1}-2 v_{i}+v_{i+1}\right) \\
\frac{\partial w_{i}}{\partial t} & =\epsilon\left(v_{i}-\gamma w_{i}\right)
\end{aligned}
$$

where $i$ runs from 1 to $n$ and the $\xi_{i}$ are Gaussian white noise terms with zero mean and unit variance. The coupling between activator components is diffusive and corresponds to the discretization of a Laplace diffusion operator. That is, this first model approximates a spatially distributed excitable medium. For the boundary elements $i=1$ and $n$ no-flux boundary conditions are used and the coupling term for $i=1$ is replaced by

$$
c\left(v_{2}-v_{1}\right)
$$

and a similar term for $i=n$.

For a numerical analysis we use $n=10$ elements. All simulations were performed with XPPAUT (Ermentrout, 2002). We chose the following parameters if not stated otherwise: $a=0.1, \epsilon=0.01$ (time scale separation), $\gamma=0.5$, $\sigma=0.02$ (noise strength), $w_{0}=-0.1$. In the original context of neural spike excitation, $w_{0}$ represents an external stimulating current. For the intracellular dynamics of calcium, this term can be associated with the stimulating effect of $\mathrm{IP}_{3}$ in the sense that a large negative value of $w_{0}$ yields a transition from an excitable regime to an oscillatory one (Atri et al., 1993). We therefore identify the increase of $\mathrm{IP}_{3}$ with a decrease of $w_{0}$. 
Without coupling, $c=0$, the individual elements exhibit the well-known excitation oscillations. Briefly, there exists a stable fixed-point which attracts trajectories from the neighborhood. For larger perturbations away from the fixed point, however, the trajectories undergo large excursions before reaching the fixed point again. In the presence of sufficiently high levels of noise in the system, the excursions, or activity spikes, occur repeatedly. They can appear similar to regular oscillations under certain conditions. Such phenomena and its characterizations have been subject of many research efforts in recent time (Lindner et al., 2004).

Turning on the coupling with strength $c>0$ leads to synchronization of the individual elements and their spikes. Three simulations with $c=0.01, c=0.1$ and $c=1$ are shown in Fig. 1. Time runs from top to bottom. For small coupling strength the elements fire in an unorganized way (a). If the coupling strength is increased waves appear (b), which become smoother with increasing coupling (c). The diffusion of activator thus leads to a strong synchronization of the individual elements. This effect is well-studied, in particular in the context of noisy media and generation of coherent signals by the coupling (Zhou et al., 2001).

This consequence of diffusion in biochemical systems is also of great importance for the $\mathrm{Ca}^{2+}$ system. As described in the introduction, calcium is released in isolated puffs or in a synchronized way. The transition from puffs to waves is typically achieved by increasing the excitability of the system, i.e., by increasing the $\mathrm{IP}_{3}$ level. But the transition to correlated activity can be reversed by addition of EGTA buffer molecules. Since EGTA is assumed to reduce the effective diffusion coefficient of $\mathrm{Ca}^{2+}$ the addition of EGTA thus acts as reduction of the coupling coefficient $c$. One can therefore have a highly responsive system (the excitability of individual channels, or clusters, is still high, as the $\mathrm{IP}_{3}$ level may be chosen large) with strongly uncorrelated activity of clusters.

In our simulations we have used two strategies to study localized and synchronized signals. One way is to prepare the system in arbitrary initial conditions and let the system evolve in an unperturbed way. The noise provides the 
necessary perturbations for repeated excitation. We then record one particular time interval showing a typical single wave or activity peak. This strategy was used to generate Fig. 1. A second strategy is to prepare the system in particular initial conditions. Here we set all variables $v_{i}, w_{i}$ to zero, which is the fixed point for $w_{0}=0$ corresponding to a small $\mathrm{IP}_{3}$ concentration. However, for $w_{0}=-0.1$ the origin is outside of the basin of attraction of the shifted fixed point. Therefore all elements will fire at least once, while the noise term is not strictly necessary. This method was used for two reasons. First, it is more easy to compare runs with different parameters, e.g. different $c$. Second, this "protocol" mimics the experimental protocol for calcium signals in oocytes. In these experiments (Dargan \& Parker, 2003) the waves are triggered by step-increasing $\left[\mathrm{IP}_{3}\right]$ (and sometimes additional forced local liberation of $\mathrm{Ca}^{2+}$ ). Most channels are thus in a rest state corresponding to the prior very low $\mathrm{IP}_{3}$ level, which is equivalent to the zero initial condition.

The conclusion from simulations with both setups is that an increase of the coupling strength, or the activator diffusion, leads to the elements fire in a synchronized way. However, closer to the main point of this study, one also finds that the local dynamics remain unchanged. This fact can be seen clearly from Fig. 2. The plot shows the evolution of a single peak for three values of $c$ and demonstrates that there is no substantial difference between the three spikes. Particularly, the three spikes last for approximately the same time (about 50 t.u.).

This observation can be understood from the following argument. In the limit of very large coupling all cells fire completely synchronized and therefore the diffusive coupling term vanishes:

$$
v_{i-1}-2 v_{i}+v_{i+1}=0, \quad(\text { complete synchronization })
$$

Accordingly, for large coupling the dynamics of an individual element is similar to the dynamics of the uncoupled element.

Furthermore, if one plots the average signal (averaged activator value $\bar{v}=$ $\sum v_{i} / 10$ ) the coupling tends to shorten the duration of the spike (data not 
shown). This happens because the firing time of each element, i.e., the time when the activator concentration starts to rise, is almost the same for all elements in the case of strong coupling, whereas for small coupling the individual firing times are spread out over a time interval. Taken together, these observations provide a strong contrast to the finding for $\mathrm{Ca}^{2+}$ waves, where a reduced coupling (higher EGTA concentration) leads to strongly shortened responses. We will therefore consider a different type of coupling in the next section.

\section{An array with time-delayed coupling}

We will now study a system of excitable elements with delayed coupling in the form:

$$
\begin{aligned}
\frac{\partial v_{i}}{\partial t}= & v_{i}\left(1-v_{i}\right)\left(v_{i}-a\right)-w_{i}-w_{0}+\sigma \xi_{i} \\
& \left.\left.+c \operatorname{Max}\left(0, v_{i-1}(t-\tau)-v_{i}\right)\right)+c \operatorname{Max}\left(0, v_{i+1}(t-\tau)-v_{i}\right)\right) \\
\frac{\partial w_{i}}{\partial t}= & \epsilon\left(v_{i}-\gamma w_{i}\right)
\end{aligned}
$$

where $i$ runs from 1 to $n$ (and no-flux boundary conditions are used at elements $i=1$ and $n$ as described in the previous section). The form of the coupling is similar to the discrete Laplacian from the previous section, but now the two activator quantities from adjacent lattice sites are delayed by a time $\tau$. We furthermore introduce the function $\operatorname{Max}(a, b)$, which gives the maximum value of $a$ and $b$ and ensures that the coupling term cannot be negative. The motivation for this function will be discussed at the end of this section.

Three simulations with $\tau=15$, and $c=0.01, c=0.1$ and $c=1$ are shown in Fig. 3 (other parameters as before). Similar to the previous case of standard diffusive coupling the elements appear to be more synchronized with increasing $c$, although the coupling may be weakened compared to the no-delay case. What is more important is that for $c=1$ the excitation persists for a much longer time (c), which means that a second time scale of signal decay is generated by the coupling. The model with delayed coupling is thus able to produce not only a smooth signal but also a much slower decay of activity for large $c$. 
To understand the underlying mechanism for the extension of spikes we will next plot the local dynamics of a single element in the phase space of activator and inhibitor. Fig. 4 shows the nullclines of an uncoupled system $(c=0)$, i.e., the curves $\partial_{t} v=0$ and $\partial_{t} w=0$. It also visualizes typical trajectories of $v_{5}$ and $w_{5}$ for small and large coupling strength. The trajectory for $c=0.02$ (small coupling) appears to be very similar to the usual relaxation spikes, that is, the trajectory jumps quickly from the fixed point to the right branch of the activator nullcline, then slowly climbs towards larger inhibition, jumps back shortly after passing the maximum value of the right nullcline branch, and travels back along the left branch. In contrast, for $c=2.0$ the trajectory continues to much larger values of the inhibitor $w$, which suggests the simultaneous stimulation from the adjacent lattice sites prevents the element to switch to lower activator levels.

To obtain figs. 3 and 4 we have used the same kind of simulation as for Fig. 1, that is, selecting for visualization a time interval of typical behavior from a much longer run. In the following we will study the responses after setting the initial values of all variables to the origin (stimulation protocol).

Fig. 5 shows the average $\bar{v}$ for four different values of $c$ using the stimulation initial conditions. The signal becomes considerably longer with increasing coupling. In terms of full duration at half maximum (FDHM) the duration extends from approximately 40 to 120 t.u.

In Fig. 6 we have plotted the FDHM for different values of time delay $\tau$. It is apparent that the largest effect occurs for time delays between 20 and 30. A comparison with Fig. 2 shows that this corresponds to about half of the spike duration. It appears that the mechanism works best if adjacent elements fire in an alternating way such that an element will fire about 50 t.u. after its neighbor has fired. The 50 t.u. consist of 25 t.u. until which a neighboring element has reached its peak activator level and the 25 t.u. of delay.

We will now discuss the significance of the $\operatorname{Max}()$ function in equation (5). We have found that the described effect, i.e., the extension of the signal, is also possible with a diffusive-delayed term: $c\left(v_{i-1}(t-\tau)-2 v_{i}+v_{i+1}(t-\tau)\right)$ if the stimulation protocol is used. In the case of a long simulation however, it 
difficult to find a regime of repeated spikes. In this case the long-time dynamics evolves towards an active rest state without spikes. This point provides no strong objection to our model, since we aim to explain experiments based on the stimulation protocol, and we can do so without the $\operatorname{Max}()$ function. As we want to use our model in the future to study repeated spiking of clusters we decided to use the model including the $\operatorname{Max}()$ function.

A further motivation for the $\operatorname{Max}()$ function originates from the details of the calcium diffusion between clusters of $\mathrm{IP}_{3}$ channels in oocytes. Since the clusters are located in a small layer close to the cell membrane, most of the $\mathrm{Ca}^{2+}$ diffuses towards the inner bulk of the cell. Only a small amount of $\mathrm{Ca}^{2+}$ diffuses along the layer towards the neighboring clusters. Therefore, if a cluster is already active and $\mathrm{Ca}^{2+}$ has accumulated locally to high concentrations (i.e. $v_{i}$ is large), the amount of $\mathrm{Ca}^{2+}$ lost toward the bulk is already large and independent on the state of the neighboring clusters. The activity of the cluster should therefore not be depressed by an inactive neighboring cluster ( $v_{i \pm 1}$ small) and in this case the coupling term should be zero. This condition is satisfied by the coupling term in equations (5). On the other hand, if a cluster is inactive (i.e. $v_{i}$ is small), the relatively small amount received by the cluster by an active adjacent cluster $\left(v_{i \pm 1}\right.$ large) can be crucial for the excitation of the cluster. In this sense, the diffusion of activating $\mathrm{Ca}^{2+}$ in the $\mathrm{IP}_{3}$ channel problem is different from the diffusion in other problems of biochemical patterning.

\section{Discussion}

We have shown in this paper, that the temporal shape of an excited pulse can be changed substantially with a time-delayed coupling. As described in the introduction, the motivation for the study comes from the existence of two time-scales in the decay of calcium release spikes. Our results provide a first evidence that the simple tuning of the cooperativity of clusters of channels might be sufficient to switch between the two different release durations. In other words, for the mechanism it is not necessary to assume a change in any of the parameters of local dynamics. 
This behavior is especially important in view of the action of slow calcium binding buffers (e.g. EGTA buffer, Dargan \& Parker (2003)). It is possible that the buffer proteins affect the local dynamics of the channels in a cluster of channels by binding calcium ions. On the other hand the buffer certainly also affects the amount of calcium that diffuses to the next cluster at a distance of a few microns. If one associates the dynamics of the single cluster with a discrete excitable element in the spirit of an FHN element, our finding implies that the action of buffer on coupling of clusters is sufficient to explain the modified time-course of calcium release upon the addition of EGTA.

On the other hand it remains to discuss a corresponding mechanism for the effect of fast $\mathrm{Ca}^{2+}$ binding protein such as the BAPTA buffer. Experimentally, the addition of BAPTA leads to a reduction of the spike amplitude and a longer release tail (Dargan \& Parker, 2003). Furthermore, due to the large reaction rates of BAPTA (around 100 times higher than EGTA) it appears possible that this buffer disrupts the communication of channels within each cluster. In the framework exploited in our approach this means that the dynamics of each excitable element is locally changed, i.e., parameters other than concerning the coupling are also changed. For instance, it is conceivable that the individual channels inside of a cluster are decoupled by reduction of $\mathrm{Ca}^{2+}$ micro-domains around the channels. Correspondingly, an element/cluster may become less excitable since a single, randomly firing channel has a reduced probability to ignite adjacent channels.

To verify our arguments we have used two different protocols of simulations. In a first protocol, we let the system run for a long time until the dynamics relaxes to a stationary behavior. If the coupling constant is large enough one still finds orchestrated activity of all elements or waves. In a second protocol we have set the initial conditions of each element to a point just below to the fixed point, outside of its basin of attraction. After starting the simulation, the trajectory goes off to the right branch of activator nullcline thus initiating a pulse. The motivation for this method is that in experiments the calcium release is stimulated by increase of $\mathrm{IP}_{3}$. $\mathrm{IP}_{3}$ concentration corresponds to one or more 
parameters of a dynamical model. The simple-most way to incorporate it into the FHN model is to associate the $\mathrm{IP}_{3}$ level with the applied current $w_{0}$. An increase of $\left[\mathrm{IP}_{3}\right]$ may shift the local fixed point such that after the $\left[\mathrm{IP}_{3}\right]$ increase the old fixed point lies outside of the basin of attraction. In that case the evolution is bound to produce the release pulse as observed in experiments and in our "stimulation" protocol. Alternatively, if $\left[\mathrm{IP}_{3}\right]$ is changed only slightly, the old state becomes to lie closer to the basin's boundary and a further fluctuation can initiate a pulse. We also note, that a repeated sequence of such $\mathrm{IP}_{3}$ increases can lead to a sequence of release pulses. This phenomenon is known as quantal release in the calcium literature (Callamaras \& Parker, 2000) and will be studied in the context of a FHN model in the future.

We finally mention that we have chosen the prototypical FHN model to demonstrate the effect of delay in the most general form. The observed change in spike form may thus also be significant for different systems such as the ryanodine receptor dynamics (Williams et al., 2007) or signal transmission between neurons (Keener \& Sneyd, 1998; Hutt \& Atay, 2005) . However, for sake of comparison with experiments on the $\mathrm{IP}_{3}$ receptor, we are now expanding this work by analysis of models that are biologically more realistic. One important example for such is model are the Li-Rinzel equations (Li \& Rinzel, 1994). We are presently analyzing both the case of delay-coupled discrete Li-Rinzel elements as well as the case of localized Li-Rinzel elements in a spatially continuous calcium bath (to be published). 


\section{References}

Atri, A., Amundson, J., Clapham, D. \& Sneyd, J. 1993. A single pool model for intracellular calcium oscillations and waves in the Xenopus laevis oocyte. Biophys.J. 65, 1727-1739.

Callamaras, N. \& Parker, I. 2000. Phasic characteristics of elementary $\mathrm{Ca}^{2+}$ release sites underlies quantal responses to $\mathrm{IP}_{3}$. The EMBO Journal, 19 (14), $3608-3617$.

Dargan, S. \& Parker, I. 2003. Buffer kinetics shape the spatiotemporal patterns of $\mathrm{IP}_{3}$-evoked $\mathrm{Ca}^{2+}$ signals. J.Physiol. 553 (3), 775-788.

Ermentrout, B. 2002. Simulating, Analyzing, and Animating Dynamical Systems. SIAM.

Falcke, M. 2004. Reading the patterns in living cells - the Physics of $\mathrm{Ca}^{2+}$ signaling. Advances in Physics, 53 (3), 255-440.

Hutt, A. \& Atay, F. 2005. Analysis of nonlocal neural fields for both general and gamma-distributed connectivities. Physica D: Nonlinear Phenomena, 203 (1-2), 30-54.

Jouaville, L., F.Ichas, Holmuhamedov, E., P.Camacho \& J.D.Lechleiter 1995. Synchronisation of calcium waves of mitochondrial substrates in Xenopus laevis oocytes. Nature, $377,438-441$.

Keener, J. \& Sneyd, J. 1998. Mathematical Physiology. Springer, New York.

Li, Y.-X. \& Rinzel, J. 1994. Equations for $\mathrm{InsP}_{3}$ receptor-mediated $\left[\mathrm{Ca}^{2+}\right]_{i}$ oscillations derived from a detailed kinetic model: a hodgkin-huxley like formalism. J.theor.Biol. 166, 461-473.

Lindner, B., Garcia-Ojalvo, J., Neimann, A. \& Schimansky-Geier, L. 2004. Effects of noise in excitable systems. Physics Reports, 392 (6), 321-424. 
Meinhold, L. \& Schimansky-Geier, L. 2002. Analytic description of stochastic calcium-signaling periodicity. Phys.Rev.E, 66, 050901-1-4.

Rüdiger, S., Shuai, J., Huisinga, W., Nagaiah, C., Warnecke, G., Parker, I. \& Falcke, M. 2007. Hybrid stochastic and deterministic simulations of calcium blips. Biophys. J. 93, 1847-1857.

Shuai, J., Pearson, J. E., Foskett, J. K., Mak, D. D. \& Parker, I. 2007. A kinetic model of single and clusterd ip3 receptors in the absence of ca2+ feedback. Biophys. J. 93, 1151-1162.

Thul, R. \& Falcke, M. 2004. Stability of membrane bound reactions. Phys.Rev.Lett. 93 (18), 188103-1-188103-4.

Williams, G., Huertas, M., Sobie, E., Jafri, M. \& Smith, G. 2007. A Probability Density Approach to Modeling Local Control of Calcium-Induced Calcium Release in Cardiac Myocytes. Biophysical Journal, 92 (7), 2311.

Yao, Y. \& Parker, I. 1995. Quantal puffs of intracellular $\mathrm{Ca}^{2+}$ evoked by inositol trisphosphate in Xenopus oocytes. J.Physiol.(Cambridge), 482 (3), 533-553.

Zhou, C., Kurths, J. \& Hu, B. 2001. Array-Enhanced Coherence Resonance: Nontrivial Effects of Heterogeneity and Spatial Independence of Noise. Physical Review Letters, 87 (9), 98101. 
Figure 1.

Space-time plots of simulations for an array with diffusive coupling of increasing strength: $c=0.01$ (a), $c=0.1$ (b), $c=1$ (c).

Figure 2.

Evolution of $v_{6}$ during a single spike for different values of coupling strength $c$ : 0.01 (dotted), 0.1 (dashed), 1.0 (solid). The gray arrows show that the duration of the spikes is unaffected by a change in the coupling constant $\left(t_{\mathrm{dur}} \approx\right.$ 26 t.u.) In other words, the strength of synchronization cannot be inferred from the local dynamics.

Figure 3.

Simulations for time-delayed coupling with different coupling strength: $c=$ $0.01(\mathrm{a}), c=0.1$ (b), $c=1$ (c). The time delay was set to $\tau=15$.

Figure 4.

Nullclines of a single, uncoupled element (gray s-shaped curve: activator; slanted line: inhibitor) and dynamics of the element $i=5$ for small and large coupling strength. For small coupling strength $(c=0.02$, dashed line) the evolution follows the usual relaxation oscillation. For large coupling strength ( $c=2$, solid line) the inhibitor grows for much longer time until a transition back to small activator levels occurs. For these simulations the noise level was set to $\sigma=0.08$.

Figure 5.

The dynamics of activator levels averaged over all 10 elements. A wave is initiated by setting both variables of all elements to zero. We show the evolution for four different values of coupling strength. Note that the peak value remains approximately constant for all $c$ values (noise level $\sigma=0.08$ ).

Figure 6 .

The full duration at half maximum time for different values of delay $\tau$. The coupling constant was set to $c=1$. Note that the strongest effect takes place 
at delay times comparable with the duration of an individual pulse (compare

Fig. 2).

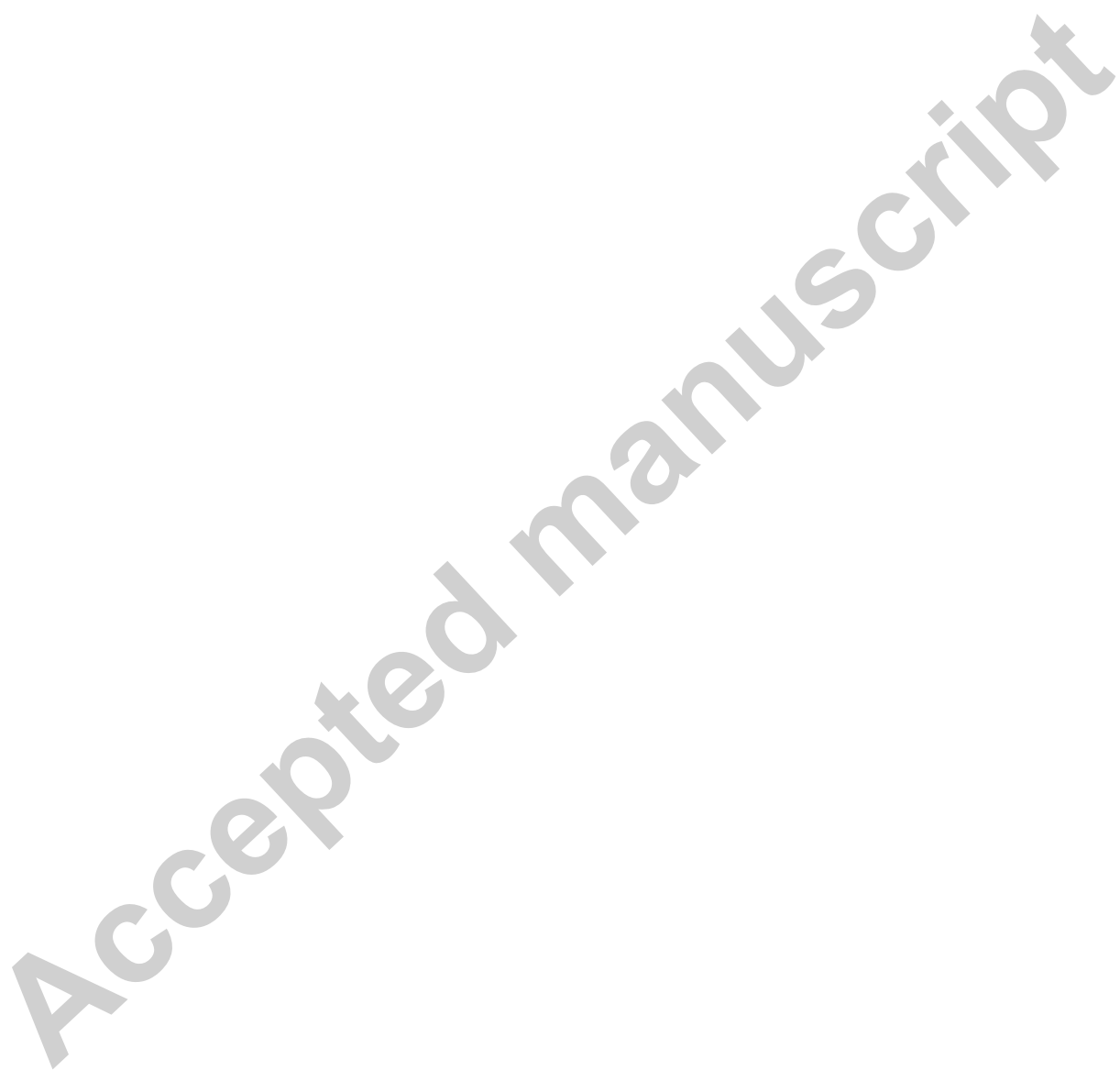


(a)

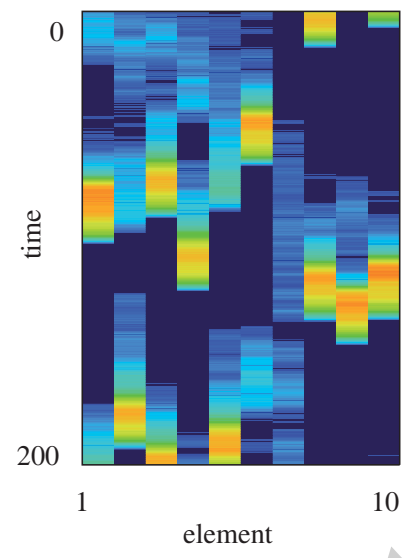

$\mathrm{v}=0$ (b)

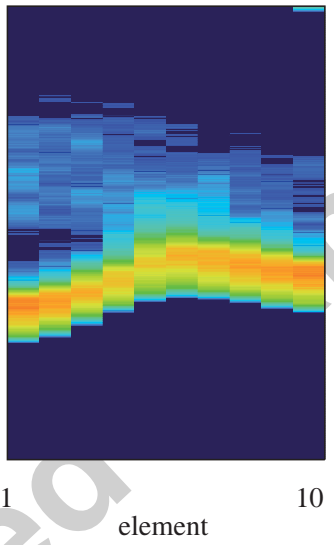

element

Figure 1: (c)

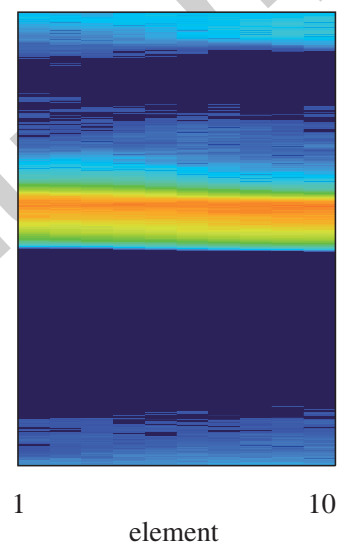

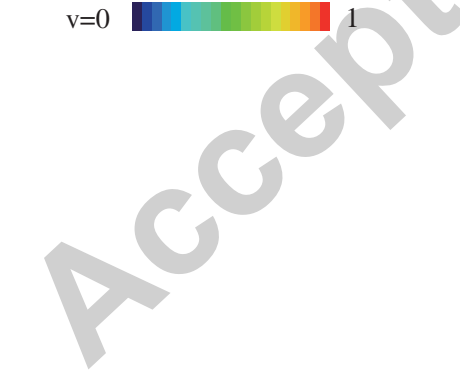




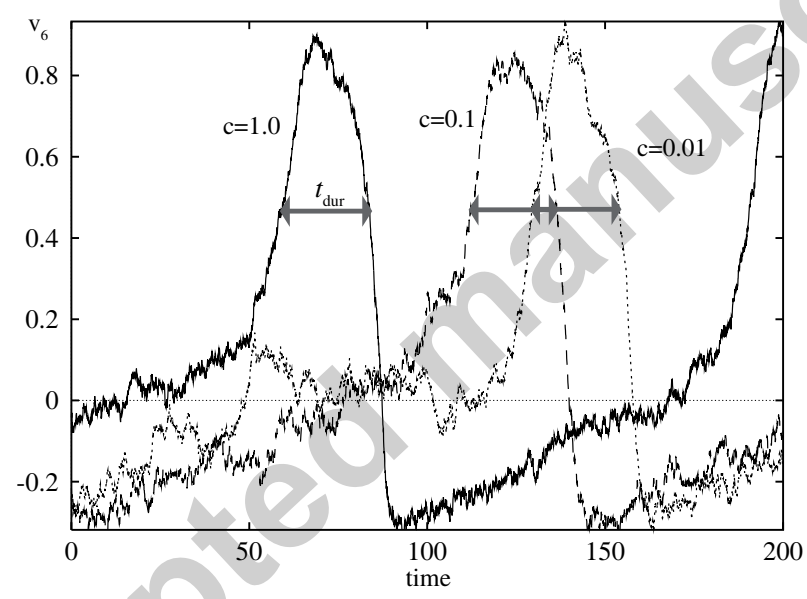

Figure 2: 
(a)

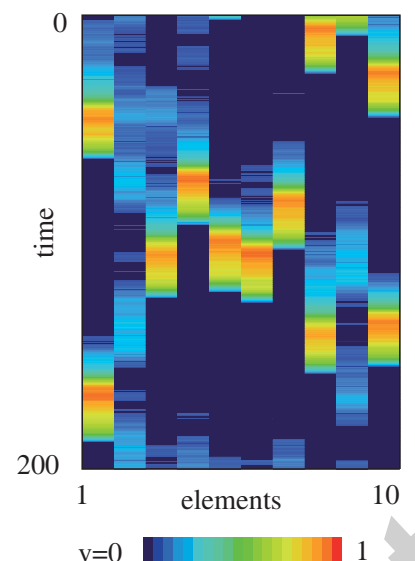

(b)

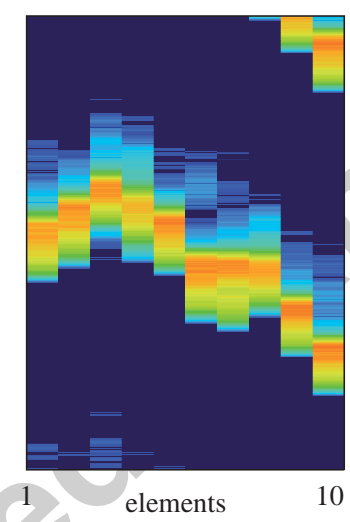

Figure 3: (c)

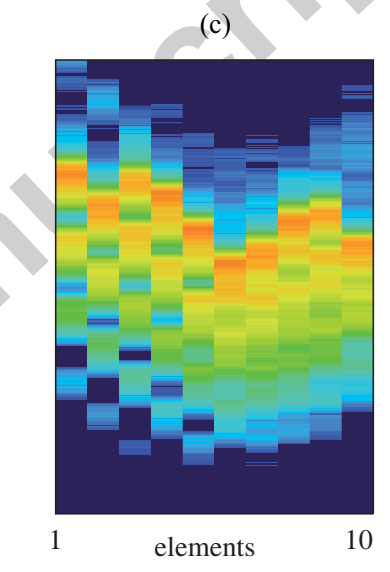

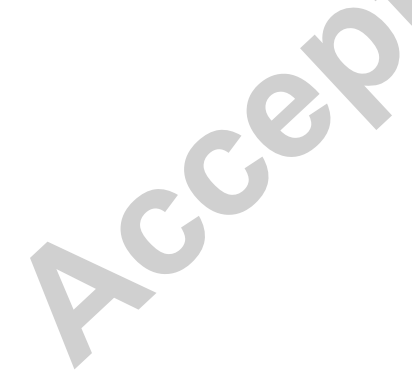




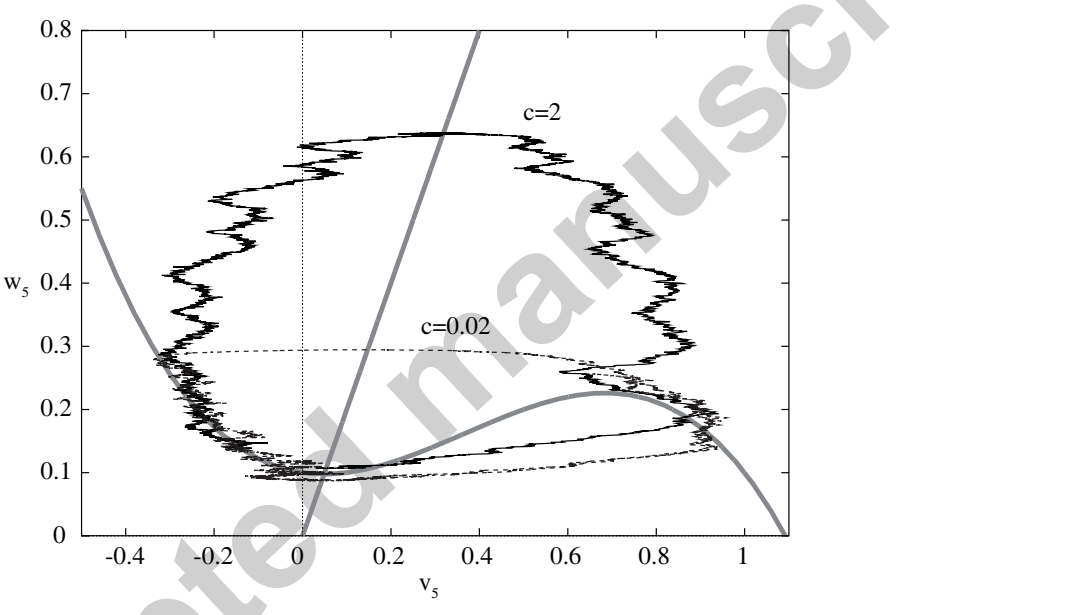

Figure 4: 


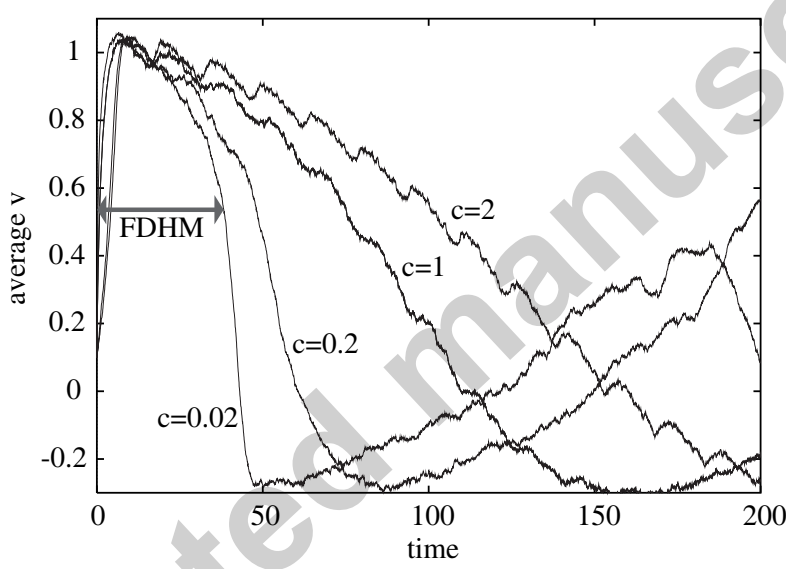

Figure 5: 


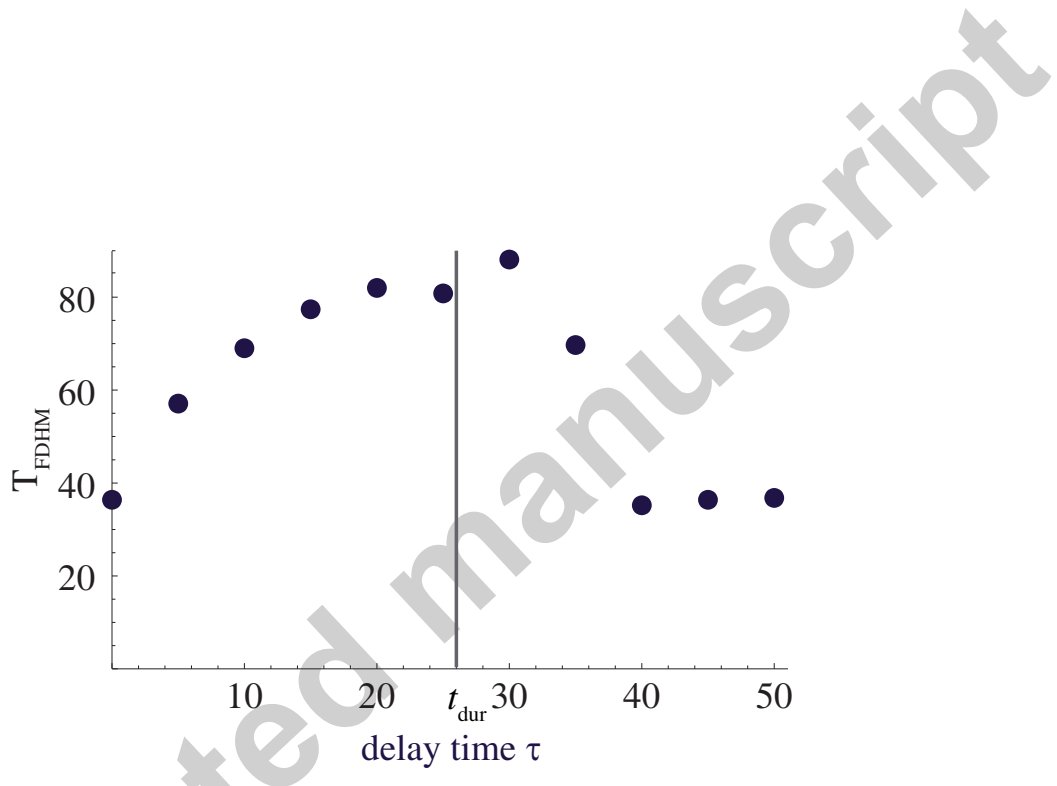

Figure 6: 